\title{
Change Detection in UWB SAR Images Based on Robust Principal Component Analysis
}

\author{
Christofer Schwartz 1,*(D), Lucas P. Ramos ${ }^{1}$, Leonardo T. Duarte ${ }^{2}{ }^{(}$, Marcelo da S. Pinho ${ }^{1}$, \\ Mats I. Pettersson ${ }^{3}{ }^{D}$, Viet T. Vu ${ }^{3}$ and Renato Machado ${ }^{1} \mathbb{D}$ \\ 1 Aeronautics Institute of Technology (ITA), São José dos Campos, SP 12228-900, Brazil; \\ lucaspr@ieee.org (L.P.R.); mpinho@ieee.org (M.d.S.P.); renatomachado@ieee.org (R.M.) \\ 2 School of Applied Sciences (FCA), University of Campinas (UNICAMP), Limeira, SP 13484-350, Brazil; \\ leonardo.duarte@fca.unicamp.br \\ 3 Blekinge Institute of Technology (BTH), 37179 Karlskrona, Sweden; mats.pettersson@bth.se (M.I.P.); \\ viet.thuy.vu@bth.se (V.T.V.) \\ * Correspondence: christofer@ieee.org; Tel.: +55-12-3947-6807
}

Received: 7 May 2020; Accepted: 10 June 2020; Published: 13 June 2020

\begin{abstract}
This paper addresses the use of a data analysis tool, known as robust principal component analysis (RPCA), in the context of change detection (CD) in ultrawideband (UWB) very high-frequency (VHF) synthetic aperture radar (SAR) images. The method considers image pairs of the same scene acquired at different time instants. The CD method aims to maximize the probability of detection (PD) and minimize the false alarm rate (FAR). Such aim fits into a multiobjective optimization problem, since maximizing the probability of detection generally implies an increase in the number of false alarms. In that sense, varying the RPCA regularization parameter leads to PD variation with respect to FAR, which is known as receiver operating characteristic (ROC) curve. To evaluate the proposed method, the CARABAS-II data set was considered. The experimental results show that RPCA via principal component pursuit (PCP) can provide a good trade-off between PD and FAR. A comparison between the results obtained with the proposed method and a classical $\mathrm{CD}$ algorithm based on the likelihood ratio test provides the pros and cons of the proposed method.
\end{abstract}

Keywords: synthetic aperture radar; CARABAS-II; RPCA; change detection; blind source separation

\section{Introduction}

Among the different approaches in unsupervised separation, robust principal component analysis (RPCA) methods can deal with additive mixing models and are commonly used for foreground detection in video surveillance systems to detect moving objects [1]. In synthetic aperture radar (SAR) applications, detecting and removing undesired content is an essential issue in practice [2]. The problem of detecting and removing content in SAR data can be treated as an unsupervised signal separation problem, usually referred to as blind source separation (BSS) [3].

Currently, the use of RPCA in SAR applications can already be found in the literature. In [4], RPCA solved via principal component pursuit (PCP) is used in a pre-processing step to decompose the SAR data into two parts, one related to stationary objects and the other to moving targets. The authors achieved separation employing a proper windowing scheme based on the data set of the X-band SAR system, namely GOTCHA Volumetric SAR. By still considering the GOTCHA data set and the RPCA via PCP, [5] presents preliminary results for multi-pass SAR change detection (CD) in X-band. In the context of ground moving target indication (GMTI), [6] proposes the use of RPCA via a relaxed version of PCP (Relaxed PCP) in order to achieve moving target detection in multichannel surveillance radar systems (MC-SAR), presenting computational results using real data from a Chinese X-band 
SAR system. Then, [7] extends the method presented in [6] aiming at clutter suppression. GMTI problem is again addressed in [8], in which a fast interferometry RPCA method is proposed considering multiple-input multiple-output (MIMO) SAR systems, where gains in terms of moving target detection and computational running time complexity are observed. Moreover, sparse representations have also been considered in other types of radars, such as in $[9,10]$. These works provided a framework based on $l_{q}$-norm constraints by means of an approach known as sparse learning via iterative minimization (SLIM).

The goal in CD applications is detecting changes in a scene that occurs between different measurement campaigns [11]. The changes can be a consequence of natural disasters or human activities (military and civilian), which result in the appearance or disappearance of targets on the ground. The challenge associated with the design of an automated CD algorithm goes beyond performing the target detection. It is also related to the clutter suppression aiming at a low false alarm rate (FAR) to provide useful information for decision making [11]. For instance, in wavelength-resolution SAR systems, such as the Coherent All Radio Band System (CARABAS) [12], false alarms are often caused by elongated structures commonly sensitive to flight path (e.g., power lines) [13].

Over the last years, CD algorithms with different approaches have been proposed aiming at higher values of probability of detection (PD) while keeping or reducing the FAR. A method similar to space-time adaptive processing (STAP), which exploits differences between target, clutter, and interference signals, was presented in [11]. STAP theory is usually applied in GMTI to combine data from different receiving channels in order to perform stationary clutter suppression and moving target detection [2]. However, in [11], the challenge is to enhance changes between two single-channel SAR images acquired at different times (image pairs). In that sense, in [11], a hypothesis test is used-which relies on a likelihood ratio test-followed by a constant false alarm rate (CFAR) normalization, thresholding, and morphological operations to reduce false detection. Testing statistics such as amplitude ratio and generalized likelihood ratio tests have been used in wavelength-resolution SAR change detection, which considers a pair of images by using the one-look data statistics $[14,15]$.

It is worth noticing that CARABAS is one of the few available UWB wavelength-resolution SAR systems. The system is operating with ultrawideband (UWB) in the VHF range (20-90 MHz), which results in low influence of speckle and high stability between measurements. That behavior allows multiple images of the same scene to have a high correlation between measurements, which, in turn, allows one to detect targets through CD algorithms [12]. Knowing that objects responsible for clutter can be considered stable for different image acquisitions once VHF UWB SAR might have only one scatter in the resolution cell for high resolution, the combination of different images taken from different flights (or from different antennas on the same device) can be used to improve the $C D$ performance. Such a feature paves the way for the use of statistical tools such as RPCA via PCP.

This paper aims to explore the use of the signal separation tool known as RPCA via PCP [16] as a $\mathrm{CD}$ algorithm where the inputs are two UWB wavelength-resolution SAR images. The issue addressed in this paper differs from the references above-mentioned in important aspects. First, the focus herein is in UWB wavelength-resolution SAR images. It can be considered as an important issue since the RPCA performance relies on the statistical behavior of the backscattered signal. In addition, detecting changes between two single-channel (SC) SAR images may present several differences in terms of signal correlation when compared to separate sparse content between MC-SAR channels for GMTI application. Thus, this paper shows that the classical model of RPCA via PCP may achieve relevant results for the class of images analyzed since the targets (or changes) are sufficiently uncorrelated from the background content. Currently, no other results are found for methods based on RPCA via PCP for this type of radar system, which has unique statistical properties. The evaluation is performed considering a methodology designed aiming at pairs of images to attend the UWB VHF SAR application which accounts for details like detections tangent between images and other measurement criteria to better 
accomplish the application. Moreover, the results presented in this paper could be considered as a baseline for other futures $C D$ algorithms proposals based on RPCA, aiming at VHF UWB SAR systems.

The rest of this paper is organized as follows. Section 2 introduces the principle of RPCA via PCP. Then, Section 3 details the materials and methods herein used. Section 4 details the proposed method to achieve RPCA via PCP change detection. Computational results are presented in Section 5. Finally, concluding remarks are given in Section 6.

\section{Robust PCA}

Principal component analysis (PCA) has been a ubiquitous tool in data analysis [17] for several decades. In order to accomplish tasks such as denoising and dimensionality reduction, PCA relies on the observation that the covariance matrix of high-dimensional tabular data sets (such as images) exhibits, very often in practice, particular structures, which means that the intrinsic dimension of a data set is usually lower than its observed dimension. As a consequence, it becomes possible to approximate high-dimension data sets by simpler structures.

In PCA, the search for a low-dimension approximation is carried out by means of the following optimization problem:

$$
\begin{array}{ll}
\text { minimize } & \|\mathbf{X}-\mathbf{L}\|_{F}^{2} \\
\text { subject to } & \operatorname{rank}(\mathbf{L}) \leq l,
\end{array}
$$

where $\mathbf{X} \in \mathbb{R}^{N \times M}$ is the observed data matrix, $\mathbf{L} \in \mathbb{R}^{N \times M}$ is the low-rank approximation of $\mathbf{X}$, and $\|\cdot\|_{F}$ denote the Frobenius norm. The rank of $\mathbf{L}$ is (at most) $l$, which is (often) lower than $\operatorname{rank}(\mathbf{X})=\min (N, M)$. In fact, the optimization problem expressed in (1) can also be seen as the problem of minimizing the Frobenius norm of a noise term $\mathbf{N}$ in the following approximation of the observed data: $\mathbf{X}=\mathbf{L}+\mathbf{N}$.

In the past decade, much effort has been put in an extension of (1) known as robust principal component analysis (RPCA) [16], mainly aiming at giving robustness to the PCA against outliers. In this case, the observed data, $\mathbf{X}$, is decomposed as follows

$$
\mathbf{X}=\mathbf{L}+\mathbf{S},
$$

where $\mathbf{L}$ corresponds to the low-rank term and $\mathbf{S}$ is a sparse matrix. Such a representation finds application in several domains, from video surveillance [1] to geophysics [18].

It is possible to perform RPCA by means of a tractable convex optimization formulation. This can be achieved by considering the nuclear norm of $\mathbf{L}$ (i.e., the sum of the singular values of $\mathbf{L}$ ), expressed by $\|\mathbf{L}\|_{*}$, as an approximation of $\operatorname{rank}(\mathbf{L})$, and the $l_{1}$ norm of $\mathbf{S}$, expressed by $\|\mathbf{S}\|_{1}$, as a measure of sparsity. Therefore, the following optimization problem can be considered

$$
\begin{array}{lc}
\operatorname{minimize} & \|\mathbf{L}\|_{*}+\lambda\|\mathbf{S}\|_{1} \\
\text { subject to } & \mathbf{L}+\mathbf{S}=\mathbf{X}
\end{array}
$$

where $\lambda>0$ is a regularization parameter that balances the two terms. An essential aspect of this formulation, which is also known as principal component pursuit (PCP), is that it can recover, under some mild conditions [16], the original terms $\mathbf{L}$ and $\mathbf{S}$ that generated the observed data according to (2).

There are several strategies to optimize (3). A very popular one is based on the alternating direction method of multipliers (ADMM) [19]. This approach can overcome a critical issue of the augmented Lagrangian method (ALM). The ALM approach is based on a single step, which means that optimization is performed by jointly considering the variables $\mathbf{L}$ and $\mathbf{S}$. On the other hand, in ADMM, the variables $\mathbf{L}$ and $\mathbf{S}$ are updated in two separate steps. The nice aspect of this approach is that each 
step in ADMM can be addressed by simpler methods. It becomes possible to set up a strategy based on alternating minimization steps [19]. In the case of RPCA, ADMM decomposes the problem as follows:

$$
\begin{gathered}
\mathbf{L}^{(t+1)}=\underset{\mathbf{L}}{\arg \min }\left\{\|\mathbf{L}\|_{*}+\frac{\rho}{2}\left\|\mathbf{L}+\mathbf{S}^{(t)}-\mathbf{X}+\rho^{-1} \Lambda^{(t)}\right\|_{F}^{2}\right\} \\
\mathbf{S}^{(t+1)}=\underset{\mathbf{S}}{\arg \min }\left\{\|\mathbf{S}\|_{1}+\frac{\rho}{2}\left\|\mathbf{L}^{(t+1)}+\mathbf{S}-\mathbf{X}+\rho^{-1} \Lambda^{(t)}\right\|_{F}^{2}\right\} \\
\Lambda^{(t+1)}=\Lambda^{(t)}+\rho\left(\mathbf{L}^{(t+1)}+\mathbf{S}^{(t+1)}-\mathbf{X}\right) .
\end{gathered}
$$

where $\rho$ represent the augmented Lagrangian parameter. The alternating steps can be solved by simply defining the shrinkage operator and shrinkage operator for singular values [19]. Due to its simplicity, the ADMM method is herein considered.

\section{Materials and Methods}

This paper proposes a CD method for UWB VHF SAR images based on RPCA via PCP. The materials and tools used for the method development and evaluation can be summarized as follows: (a) the CARABAS-II data set of images, (b) an RPCA via PCP algorithm implementation, (c) a classical CD algorithm based on the likelihood ratio test.

CARABAS-II is a VHF low-frequency SAR UWB device and represent the second generation of Swedish CARABAS radars, developed by the company Saab AB. A data set composed by 24 images achieved using the CARABAS-II system is documented in [11], and made available as an open data set by the U.S. Air Force Research Lab (AFRL) at the sensor data management system (SDMS) website [20].

More precisely, the SAR raw data were recorded into onboard hard drives leaving all signal processing steps to be performed offline, such as image formation, radiometric calibration, geocoding, equalization, and others [12]. The 24 images are georeferenced according to the Swedish reference system RR92 [21], and show the same ground area in a restricted military zone, differing from each other in target positions (missions) and flight-heading of its acquisition (passes). The final images can be considered as the results of contributions from different sources (e.g., targets, background, and additive noise). In fact, this is a superposition process in the complex domain, where the final SAR images contain amplitude and phase information. However, in the data set provided at [20], the phase information has been removed by taking the absolute value of the data. Thus, all the formulation presented in this paper is considering the SAR magnitude images (real positives).

Each magnitude SAR image covers an area of $2 \mathrm{~km}$ per $3 \mathrm{~km}$. For the CARABAS-II, the spatial resolutions are $2.5 \mathrm{~m}$ in azimuth and also in range, as reported in [22]. With the image pixel of the SAR images of $1 \mathrm{~m}$ in azimuth and range [13]. Thus, each image is composed by 6 million pixels, organized in a matrix of 3000 rows per 2000 columns. More information about the CARABAS-II system parameters can be found in [11].

For wavelength-resolution SAR systems such as CARABAS, the wavelength is larger than the resolution cell $[11,15]$. Since there is only a single scatterer per resolution cell, and the wavelength at the center frequency is in the order of a few meters, small objects are poorly reflected. This property makes the system callousness to soil roughness and forest canopy, as well as the presence of shrubs, leaves, small branches, among other objects of similar nature and scale. Distinct reflections come from objects that are placed some meters above the ground and have dimensions of meters. Hence, reflections regarding elongated structures and large dimensions objects are very sensitive to the flight path, as well as their sidelobes. Additionally, scatters of a few meters or higher do not change between measurements, which makes the noise resulting from speckles low enough, even for time intervals of several hours, days, or months.

For instance, Figure 1 brings two amplitude SAR images acquired in different timestamps, where the behavior mentioned above can be observed. The bright objects are vehicles deployed under the concealment of the foliage in the forest, which can be identified by the arrays of bright dots in 
the upper-left (mission 2) of Figure 1a and lower-right (mission 5) of Figure 1b. Both acquired with the same flight-heading (pass 3) [11]. Other bright parts are related to structures such as power lines, boulders, and fences.

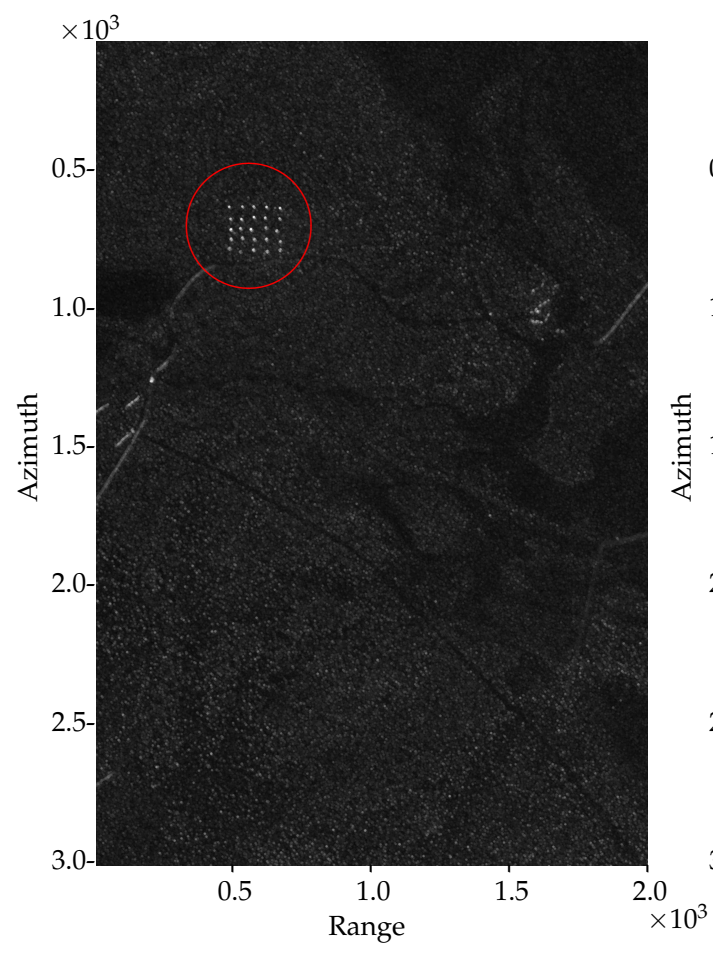

(a)

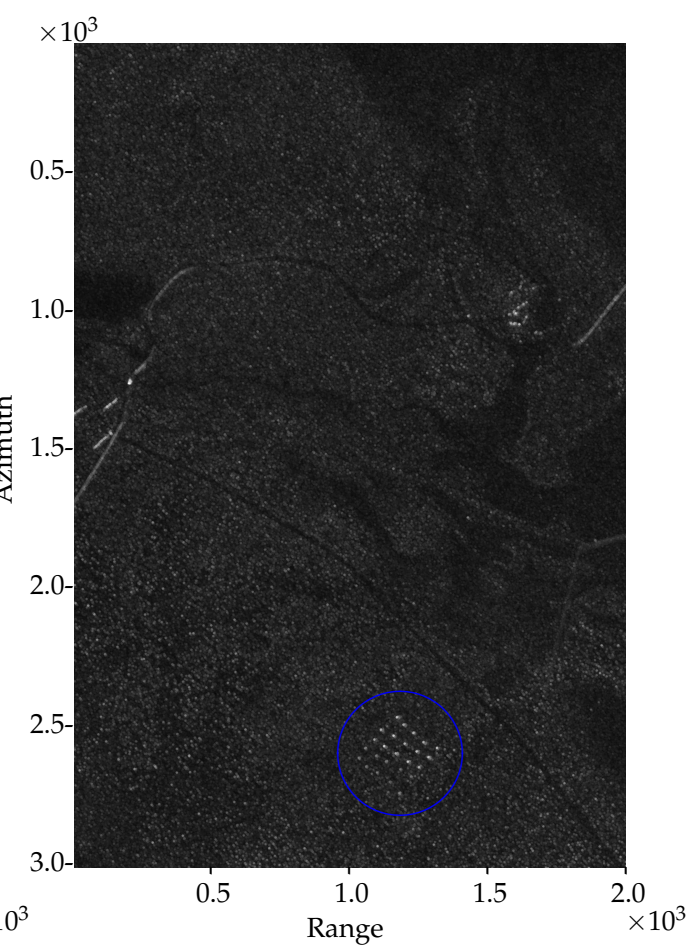

(b)

Figure 1. Magnitude SAR images of two different deployments. (a) Mission 2, Pass 3, (b) Mission 5, Pass 3. Morphological operation (one dilate) was used here to favor the visualization of the targets in this figure.

Following with the description of the materials and methods, the $\mathrm{CD}$ method herein proposed makes use of a MATLAB implementation of the RPCA via PCP available at [23]. Indeed, [23] provides a MATLAB implementation of the RPCA via PCP algorithm, whose formulation was presented in the Section 2.

The results achieved with the proposed method-which will be detailed in Section 4-are compared to the ones obtained with the classical $C D$ algorithm based on the likelihood ratio test [11]. The algorithm described in [11] was implemented with MATLAB, and the runtime analysis was made using the same processing platform for both $\mathrm{CD}$ algorithms. The processing platform is composed by a Intel Core i7-3610QM processor, operating with $8 \mathrm{~Gb}$ of RAM, using a solid state hard drive.

\section{RPCA Evaluation Method}

Let $\mathcal{I}_{m}$ be an image of a scene measured at a given measurement $m$, formed by an array of magnitudes of $R$ rows and $C$ columns. A separation model can be considering $\mathcal{I}_{m}$ as the sum of three matrices, given by

$$
\mathcal{I}_{m}=\mathcal{B}_{m}+\mathcal{T}_{m}+\mathcal{N}_{m}
$$

where $\mathcal{B}_{m}$ denotes the background content, $\mathcal{T}_{m}$ a matrix containing the targets, and $\mathcal{N}_{m}$ a matrix of additive noise.

Generally, the CD problem between two SC SAR images is based on an image-difference formulation. This is especially true for wavelength-resolution SAR systems like CARABAS, due to its backscatter characteristics [14,15]. Thus, multiplicative noise can be ignored and, as a consequence, the $\mathrm{CD}$ does not need to be based on rationing. Considering that issue, detecting changes between measurements 1 and 2 can be performed by $\mathcal{I}_{1}-\mathcal{I}_{2}$. Then, if the background is unchanged during the 
data collections (i.e., $\mathcal{B}_{1}=\mathcal{B}_{2}$ ), which is true for wavelength-resolution SAR, it is possible to retrieve the positive differences, $T_{1}$, and the negative differences, $T_{2}$, by

$$
\mathcal{I}_{1}-\mathcal{I}_{2}=\mathcal{T}_{1}-\mathcal{T}_{2}+\mathcal{N}_{1-2},
$$

where, $\mathcal{N}_{1-2}$ is a Gaussian noise term resulting from $\mathcal{N}_{1}-\mathcal{N}_{2}$ [15].

In this paper, that issue is addressed in a different manner. Let $\mathcal{V}_{1}$ and $\mathcal{V}_{2}$ be the images $\mathcal{I}_{1}$ and $\mathcal{I}_{2}$ transformed into the vector form, respectively. Similarly to the RPCA video surveillance application, $\mathcal{V}_{1}$ and $\mathcal{V}_{2}$ can be organized in the observed data matrix, $\mathbf{X}$, as

$$
\mathbf{X}=\left[\begin{array}{l}
\mathcal{V}_{1} \\
\mathcal{V}_{2}
\end{array}\right]=\left[\begin{array}{llll}
p_{1} & p_{2} & \ldots & p_{R \times C} \\
\hat{p}_{1} & \hat{p}_{2} & \ldots & \hat{p}_{R \times C}
\end{array}\right]
$$

where $p_{1}, \ldots, p_{R \times C}$ and $\hat{p}_{1}, \ldots, \hat{p}_{R \times C}$ represent the magnitude values of image pixels on vectors $\mathcal{V}_{1}$ and $\mathcal{V}_{2}$, respectively. Thus, for the CARABAS-II image set, $\mathbf{X}$ has two rows and $6 \times 10^{6}$ columns, that is, $N=2$ and $M=R \times C$.

Considering the RPCA statements presented in Section 2, it is expected to decompose the observed matrix $\mathbf{X}$, into a sparse matrix $\mathbf{S}$, and a low-rank matrix $\mathbf{L}$, keeping into the matrix $\mathbf{S}$ the content that most diverges between the two rows of matrix $\mathbf{X}$, that is, the changes between measurements 1 and 2 . This expected result is supported by the relative background stability presented by wavelength-resolution SAR system. Due to $N=2$ and $N<<M$, the rank of $\mathbf{L}$ may remain equal to the rank of $\mathbf{X}$ for several values of $\lambda$. The separation of content from $\mathbf{L}$ to $\mathbf{S}$ is performed during the iterations of the RPCA algorithm until achieving a given value of sparsity to $\mathrm{S}$. This target value of sparsity relies on the $\lambda$ value. Lower values of $\lambda$ result in more information in $\mathbf{S}$ (non-zero values), which increases the PD and FAR. The content that will be priorly transferred to $\mathbf{S}$ depends on the correlation structure of the input matrix. Hence, it is possible to balance the content of the matrix $\mathbf{S}$ such that the targets are contained in $\mathbf{S}$, avoiding the noise and false alarms contents whenever possible. In that sense, sparse content with respect to the images $\mathcal{I}_{1}$ and $\mathcal{I}_{2}$ will be distributed, respectively, in the first and second rows of matrix $\mathbf{S}$.

\section{Measurement Criteria}

Each non-zero value in $\mathbf{S}$ is considered a detection. Similarly to [11], the targets are considered detected if detections exist within a radius of $10 \mathrm{~m}$ (i.e., 10 pixels) from the ground truth position. Defining PD as the number of detected targets divided by the number of known targets, it is possible to calculate an average PD for a given amount of CD tasks. Other detections that could not be related to any target is counted as false alarms. After counting, the FAR is calculated as the number of false alarms per square kilometer.

In this paper, the false alarms are counted by using a window of $10 \times 10$ pixels. For instance, ten consecutive pixels (in one or both directions) are considered with respect to the same object and counted as one false alarm. Ten distant pixels are considered distinct objects and counted as ten false alarms. Following that rule, for example, 100 consecutive pixels will be counted as ten false alarms and 100 separate pixels as 100 false alarms. This metric allows a better ranging of the RPCA $\lambda$ parameter by dealing with lower values, which increases the number of false alarms that are related to the same objects.

Also, the proposed method allows decision making in the following singular situation. When detections related to false alarms exist within the window of $10 \times 10$ pixels on both rows of matrix S. This may be the case if an object moves a few meters within the window or due to scattering variations between measurements. Since variations within the window of $10 \times 10$ pixels can be related to the same object, such cases will not be considered changes and will be counted separately as detections tangent between rows (DTBR). More details about DTBR is given in Section 5. 
It is worth mentioning that $\mathrm{CD}$ algorithms which make use of morphological operations naturally associate adjacent pixels to the same object. The proposed method does not use CFAR normalization, threshold, or any morphological operations (e.g., erode, dilate). Therefore, CD performance depends only on the chosen $\lambda$ value.

\section{Results}

The default formulation presented in [16] to calculate the $\lambda$ value is given by $\lambda=(\sqrt{R \times C})^{-1}$ for $N<M$ and $M=R \times C$. This results in $\lambda=4.082 \times 10^{-4}$ for the CARABAS-II data set. However, the choice of a suitable $\lambda$ value relies on the data set statistical behavior in addition to the input matrix dimensions. Therefore, a workable operating region was found empirically by ranging the $\lambda$ value. More specifically, an average PD, FAR, and DTBR, were calculated for each evaluated $\lambda$ value, considering the 24 image pairs defined in [11]. Then, a workable operating region was observed within the range of 6 to 13 times the value of $4.082 \times 10^{-4}$.

Figure 2a shows the results for the following $\lambda$ values: $0.0024,0.0029,0.0033,0.0037,0.0041,0.0045$, 0.0049 , and 0.0053 . It is possible to observe that as the $\lambda$ value increases within the range, the PD and FAR are affected differently. More precisely, for $\lambda=0.0024$, an average PD of 0.985 can be achieved while an undesirable FAR of 6.583 is obtained. However, if a PD above 0.95 is considered acceptable, better relationships between PD and FAR may be achieved for $\lambda$ values of 0.0029 to 0.0033 . Moreover, $\lambda=0.0037$ can be an option to keep the FAR lower than 1 , aiming at a reasonable PD of 0.8850 . The other $\lambda$ values may be considered in circumstances in which lower values of FAR is needed. It is worth observing that FAR related to DTBR is accounted for separately. As mentioned in Section 4, DTBR can be considered null but used for decision making on stringent applications. One case of DTBR is illustrated in Figure 3a at azimuth 1000-1500, range 0-500, marked with two overlapping circles in red and blue colors, respectively.

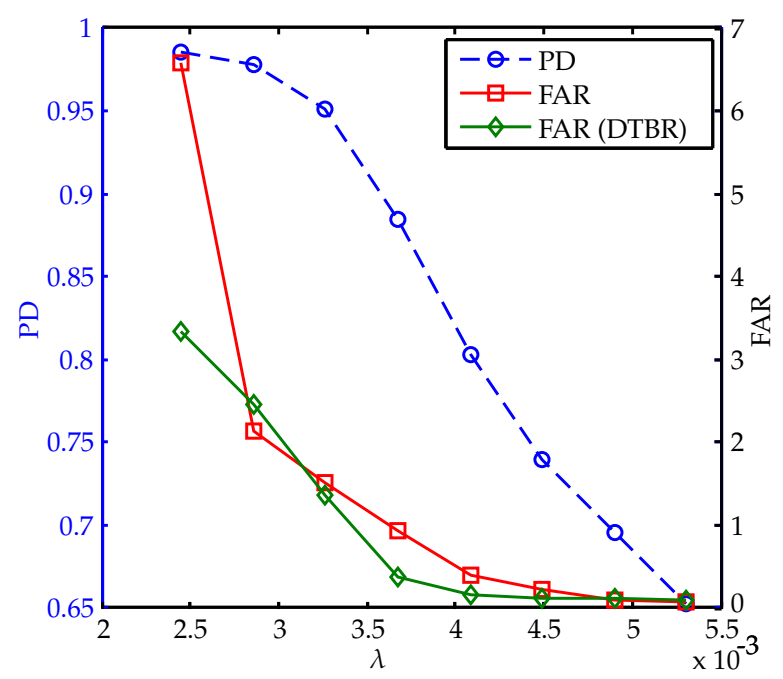

(a)

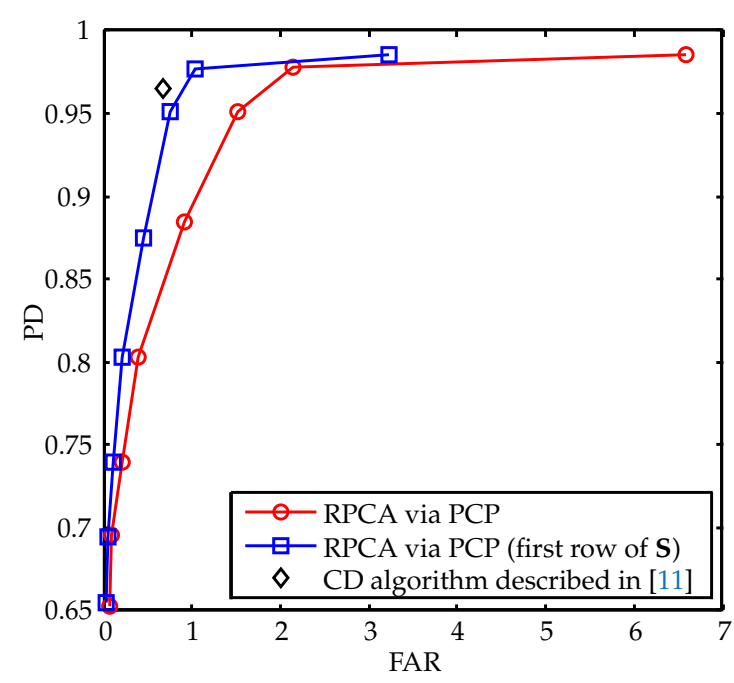

(b)

Figure 2. Performance analysis curves: (a) Average values of PD (left axis), FAR, and DTBR (right axis), for the $\lambda$ values within the workable operating region. (b) Receiver operating characteristic curve. 


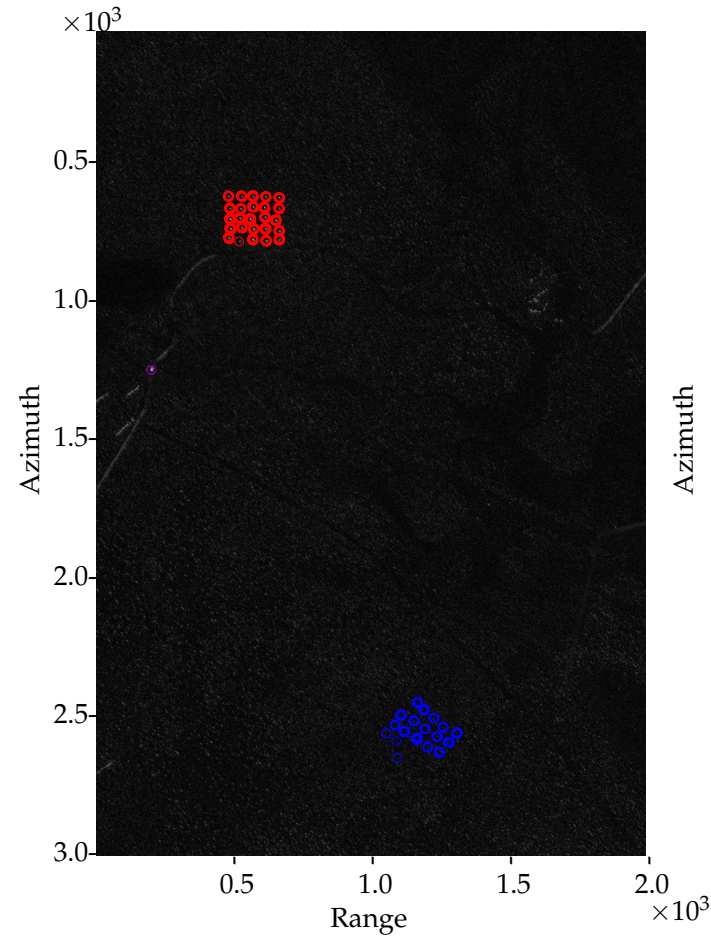

(a)

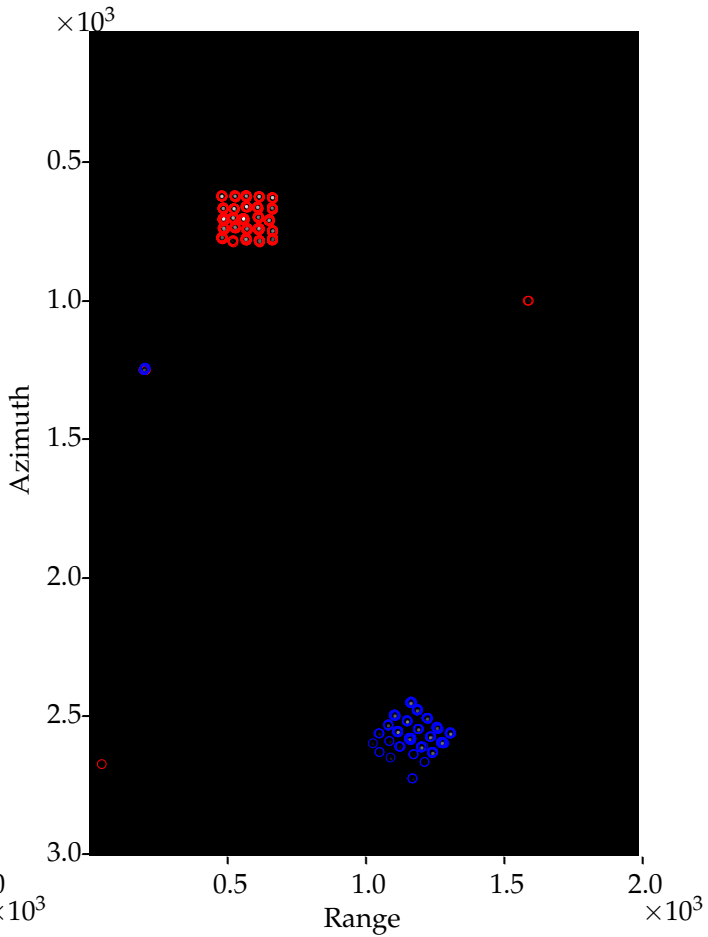

(b)

Figure 3. CD results for the image pair formed with the amplitude SAR images of Mission 2, Pass 3, and Mission 5, Pass 3: (a) for $\lambda=0.0037$ and (b) for $\lambda=0.0033$. Mission 2, Pass 3, is also used as background-image in (a).

Besides the DTBR, Figure 3a shows the CD results for $\lambda=0.0037$ using an image pair formed with the amplitude SAR images of Mission 2, Pass 3, as $\mathcal{I}_{1}$, and Mission 5, Pass 3, as $\mathcal{I}_{2}$. Each detection is automatically marked with circles. Detections related to $\mathcal{I}_{1}$ are observed between azimuth 500-1000 and around range 500. Similarly, the circles related to $\mathcal{I}_{2}$ are observed around azimuth 2500 , between range $1000-1500$. Note that $\mathcal{I}_{1}$ is also used as a background-image in Figure 3a for guidance purposes. Thus, the circles related to $\mathcal{I}_{2}$ are not accompanied by the array of bright dots. The introduced method detected 43 of 50 targets, specifically, 25 targets related to $\mathcal{I}_{1}$ and 18 targets related to $\mathcal{I}_{2}$. Since the PD is calculated by the ratio of detected vehicles to the total number of vehicles, then $\mathrm{PD}=43 / 50=0.86$. Once no false alarms are observed, FAR $=0$ considering the DTBR null. Figure $3 \mathrm{~b}$ shows the results for $\lambda=0.0033$ to illustrate the pros and cons in decreasing the $\lambda$ value. It is possible to observe an increase in detected changes related to $\mathcal{I}_{2}$, while two false alarms appeared.

Another approach to calculate the PD and FAR can be considering only the detections related to one of two images. This allows comparing the results with $\mathrm{CD}$ algorithms that play the role of detecting targets only in the surveillance-image. In that sense, if $\mathcal{I}_{1}$ is the surveillance-image, and $\mathcal{I}_{2}$ is considered only as the reference-image, then detections associated with the second row of matrix $\mathbf{S}$ might be discarded, except for the DTBR analyzes. Therefore, PD can be calculated as PD $=25 / 25=1$, keeping no false alarms. Thus, the obtained results can be better compared, for instance, to the ones obtained by the classical CD algorithm described in [11], which reached in PD $=25 / 25=1$, and FAR $=3 / 6=0.5$, using $\mathcal{I}_{1}$ and $\mathcal{I}_{2}$ as surveillance-image and reference-image, respectively.

General comparison considering the 24 image pairs can be made by observing Figure $2 b$. Actually, Figure $2 b$ shows the receiver operating characteristic (ROC) curve of the RPCA via PCP method. Additionally, Figure $2 \mathrm{~b}$ presents the ROC curve considering only the detections related to the first row of matrix $\mathbf{S}$. The point indicating the average performance of the CD algorithm in [11] is also provided for comparison, which is given by $\mathrm{PD}=0.965$ and FAR $=0.667$. It is possible to observe that the proposed method obtained similar performance for $\lambda=0.0033$, when compared to the CD algorithm in [11]. In fact, $\mathrm{PD}=0.952$ and $\mathrm{FAR}=0.763$, which means a slightly lower performance if operating 
in $\mathrm{PD}=0.965$ is required. Alternatively, $\mathrm{PD}=0.976$ can be reached if an increase in FAR of 0.36 is allowed. Reduce the FAR to 0.444 is also an option if PD $=0.875$ is sufficient.

\section{Discussion}

This paper proposed a method based on RPCA via PCP to perform CD in wavelength-resolution SAR images. The method considers the use of image pairs provided by a VHF UWB SAR system, and are evaluated using the CARABAS-II challenged data set, available at U.S. Air Force SDMS website. The results indicate that RPCA via PCP can be used for UWB VHF SAR change detection applications. More precisely, original results illustrate the merits of the RPCA via PCP as a CD method for the wavelength-resolution SAR system. Moreover, contributions regarding the method, which account details like the DTBR and other measurement criteria, can also be observed. Thus, the results presented in this paper could be considered for future solutions that can be developed from the proposed method.

Despite the similar performance for $\lambda=0.0033$, the proposed method presents several gains in terms of runtime processing. Specifically, the proposed method spent an average time of $54 \mathrm{~s}$ to each CD task, while the CD algorithm in [11] (also implemented in MATLAB to comparison) required a runtime about 10 times longer for the same processing platform. Both implementations considering single-thread execution. This behavior can be explained by the differences in the mathematics behind both $C D$ strategies. In fact, a matrix solution may present several advantages in terms of runtime processing when compared to a pixel by pixel solution. The RPCA MATLAB implementation available in [23] uses the singular value decomposition (SVD) in economy size mode. This mode computes only the first $N$ columns of the singular vectors when the number of rows is lower than the number of columns. In this case, singular values, returned as a diagonal matrix, is an $N$ by $N$ matrix. This condition shown to be sufficient, since no gains in terms of detection were observed in using the transpose of $\mathbf{X}$ as input.

It is worth mentioning that many efforts have been made in developing algorithms to solve RPCA via PCP aiming at computational reductions. In [1], different algorithms are listed and classified as: basics, linearized, and fast algorithms. Moreover, [1] also describes algorithms that require computing the SVD results in $\mathcal{O}(M \times N \times \min (M, N))$ complexity. That issue motivates researches focusing on the reduction of the complexity by avoiding the computation of SVD. Thus, it is expected that the use of fast algorithms will present even more reductions in runtime. More information about such algorithms can be found in [1].

Furthermore, some individual comparison can be made. Using $\lambda=0.0037$ as an example, the proposed method presents better results for six image pairs. FAR reductions are also observed to other seven image pairs but with lower PD values. Equal and worse results are observed for two and nine image pairs, respectively. This behavior can be explained by the differences in the mathematics behind both CD strategies. This issue can be considered on developing algorithms combining different mathematical strategies to better explore the images features.

The results achieved in this study motivate the development of other methodologies for UWB VHF SAR change detection, based in other sparse representations. For instance, an approach based on a convex solution would be very welcome [24]. Thus, such challenges can be addressed in future studies.

Author Contributions: Conceptualization: C.S., L.T.D. and R.M.; methodology: C.S. and L.P.R.; software: C.S. and L.P.R.; validation: C.S., L.P.R., L.T.D., M.d.S.P., M.I.P., V.T.V. and R.M.; formal analysis: L.T.D., M.d.S.P. and R.M.; investigation: C.S., L.P.R., L.T.D., M.d.S.P., M.I.P., V.T.V. and R.M.; resources: L.T.D., M.I.P., V.T.V. and R.M.; writing-original draft preparation: C.S., L.P.R., and L.T.D.; supervision: L.T.D., M.d.S.P., M.I.P., V.T.V. and R.M.; funding acquisition: C.S., R.M., L.T.D., M.I.P.; project administration: C.S., R.M.; All authors have read and agreed to the published version of the manuscript.

Funding: This study was financed in part by the Coordenação de Aperfeiçoamento de Pessoal de Nível Superior-Brazil (CAPES-Brazil)_Finance Code 001 (Pró-Defesa IV) and through the scholarship PNPD-CAPES grant 88882.316964/2019-01 (Corresponding author C.S.). The authors also thank the Conselho Nacional de Desenvolvimento Científico e Tecnológico (CNPq-Brazil) for partial financial support, grant 311357/2017-2 (Corresponding author L.T.D.). 
Acknowledgments: The authors would like to thank the Swedish Defence Research Agency (FOI) and Saab AB for support and the U.S. Air Force Research Lab (AFRL) for the open data set.

Conflicts of Interest: The authors declare no conflict of interest. The funders had no role in the design of the study, no role in the collection, analyses, or interpretation of data, and no role in the writing of the manuscript, or in the decision to publish the results.

\section{References}

1. Bouwmans, T.; Zahzah, E.H. Robust PCA via principal component pursuit: A review for a comparative evaluation in video surveillance. Comput. Vis. Image Underst. 2014, 122, 22-34. [CrossRef]

2. Klemm, R. Principles of Space-Time Adaptive Processing, 3rd ed.; Radar, Sonar \& Navigation, no. 21; Institution of Engineering and Technology: London, UK, 2006. [CrossRef]

3. Comon, P.; Jutten, C. Handbook of Blind Source Separation-Independent Component Analysis and Applications, 1st ed.; Academic Press: Cambridge, MA, USA, 2010. [CrossRef]

4. Borcea, L.; Callaghan, T.; Papanicolaou, G. Synthetic Aperture Radar Imaging and Motion Estimation via Robust Principal Component Analysis. SIAM J. Imaging Sci. 2013, 6, 1445-1476. [CrossRef]

5. Hu, Z.; Bryant, M.; Qiu, R.C. Multi-path SAR change detection. In Proceedings of the 2012 IEEE Radar Conference, Atlanta, GA, USA, 7-11 May 2012; pp. 0859-0863. [CrossRef]

6. Yan, H.; Wang, R.; Li, F.; Deng, Y.; Liu, Y. Ground Moving Target Extraction in a Multichannel Wide-Area Surveillance SAR/GMTI System via the Relaxed PCP. IEEE Geosci. Remote Sens. Lett. 2013, 10, 617-621. [CrossRef]

7. Yang, D.; Yang, X.; Liao, G.; Zhu, S. Strong Clutter Suppression via RPCA in Multichannel SAR/GMTI System. IEEE Geosci. Remote Sens. Lett. 2015, 12, 2237-2241. [CrossRef]

8. Huang, Y.; Liao, G.; Xu, J.; Li, J.; Yang, D. GMTI and Parameter Estimation for MIMO SAR System via Fast Interferometry RPCA Method. IEEE Trans. Geosci. Remote Sens. 2018, 56, 1774-1787. [CrossRef]

9. Tan, X.; Roberts, W.; Li, J.; Stoica, P. Sparse Learning via Iterative Minimization With Application to MIMO Radar Imaging. IEEE Trans. Signal Process. 2011, 59, 1088-1101. [CrossRef]

10. Aubry, A.; Carotenuto, V.; Maio, A. De; Govoni, M. A. Multi-Snapshot Spectrum Sensing for Cognitive Radar via Block-Sparsity Exploitation. IEEE Trans. Signal Process. 2019, 67, 1396-1406. [CrossRef]

11. Lundberg, M.; Ulander, L.M.H.; Pierson, W.E.; Gustavsson, A. A challenge problem for detection of targets in foliage. Proc. SPIE 2006, 6237, 1-12. [CrossRef]

12. Ulander, L.M.; Froelind, P.O.; Gustavsson, A.; Hellsten, H.; Larsson, B. Detection of concealed ground targets in CARABAS SAR images using change detection. Proc. SPIE 1999, 3721, 243-252. [CrossRef]

13. Vu, V.T.; Pettersson, M.I.; Machado, R.; Dammert, P.; Hellsten, H. False Alarm Reduction in WavelengthResolution SAR Change Detection Using Adaptive Noise Canceler. IEEE Trans. Geosci. Remote Sens. 2017, 55, 591-599. [CrossRef]

14. Ulander, L.M.H.; Lundberg, M. Modeling of change detection in VHF- and UHF-band SAR. In Proceedings of the 7th European Conference on Synthetic Aperture Radar, Friedrichshafen, Germany, 2-5 June 2008; pp. 1-4.

15. Machado, R.; Vu, V.T.; Pettersson, M.I.; Dammert, P.; Hellsten, H. The Stability of UWB Low-Frequency SAR Images. IEEE Geosci. Remote Sens. Lett. 2016, 13, 1114-1118. [CrossRef]

16. Candès, E.J.; Li, X.; Ma, Y.; Wright, J. Robust Principal Component Analysis? J. ACM 2011, 58, 1-37. [CrossRef]

17. Udell, M.; Horn, C.; Zadeh, R.; Boyd, S. Generalized low rank models. Found. Trends Mach. Learn. 2016, 9, 1-118. [CrossRef]

18. Duarte, L.T.; Nadalin, E.Z.; Filho, K.N.; Zanetti, R.A.; Romano, J.M.T.; Tygel, M. Seismic wave separation by means of robust principal component analysis. In Proceedings of the 20th European Signal Processing Conference (EUSIPCO), Bucharest, Hungary, 27-31 August 2012; pp. 1494-1498.

19. Boyd, S.; Parikh, N.; Chu, E.; Peleato, B.; Eckstein, J. Distributed Optimization and Statistical Learning via the Alternating Direction Method of Multipliers. Found. Trends Mach. Learn. 2011, 3, 1-122. [CrossRef]

20. U.S. Air Force. The Sensor Data Management System. Available online: https://www.sdms.afrl.af.mil (accessed on 2 December 2019). 
21. Walter, F.; Fransson, J.E.S.; Frolind, P.O. Fully automatic geo-coding of CARABAS-II VHF SAR images. In Proceedings of the IGARSS'99, Hamburg, Germany, 28 June-2 July 1999; Volume 1, pp. 569-573. [CrossRef]

22. Ulander, L.M.H.; Lundberg, M.; Pierson, W.; Gustavsson, A. Change detection for low-frequency SAR ground surveillance. IEE Proc.-Radar Sonar Navig. 2005, 152, 413-420. [CrossRef]

23. Laptev, D. Robust PCA. Available online: https://github.com/dlaptev/RobustPCA (accessed on 2 December 2019).

24. Lin, C.H.; Wu, R.; Ma, W.K.; Chi, C.Y.; Wang, Y. Maximum Volume Inscribed Ellipsoid: A New Simplex-Structured Matrix Factorization Framework via Facet Enumeration and Convex Optimization. SIAM J. Imaging Sci. 2018, 11, 1651-1679. [CrossRef]

(C) 2020 by the authors. Licensee MDPI, Basel, Switzerland. This article is an open access article distributed under the terms and conditions of the Creative Commons Attribution (CC BY) license (http:/ / creativecommons.org/licenses/by/4.0/). 